\title{
Case report of Schizophyllum communesinusitis in an immunocompetent patient
}

\author{
Luz Ángela C astro, Bact ${ }^{1}$, M aríalnés Álvarez, B act, MSc² ${ }^{2}$ ErnestoMartínez , MD³ \\ SUMMARY
}

Schizophyllum commune is a basidiomycete fungus with broad distribution in nature; however, it is a rare cause of infectious disease. We report the isolation of this mould in a 46 year-old immunocompetent patient with chronic sinusitis previously treated with multiple antibiotics and topical nasal steroids. Material obtained via a left maxillary sinus antrostomy showed septate hyaline hyphae with clamp connections on direct examination with $\mathrm{KOH}$ and histopathological studies. Further growth on Sabouraud agar produced a white mould that, based on its microscopic and macroscopic characteristics, was identified asS. commune. Despite its low frequency, this fungus should be considered a possible pathogen, particularly in samples obtained from paranasal sinuses.

Keywords: Basidiomycete; Sinusitis; Schizophyllum commune; Diagnostic; Itraconazole.

\section{Informe de un caso de sinusitis por Schizophyllum commune en enfermo inmuno-competente}

\section{RESUMEN}

Schizophyllum commune es un hongo basidiomiceto con una amplia distribución en la naturaleza, que con poca frecuencia causa enfermedad infecciosa. Se informa el aislamiento de este moho, en un paciente inmuno-competente de 46 años con sinusitis crónica tratada previamente con múltiples antibióticos y esteroides tópicos nasales. Por una antrostomía maxilar izquierda se obtuvo una muestra que en el examen directo con $\mathrm{KOHy} \mathrm{tinciones} \mathrm{histopatológicas,} \mathrm{mostró} \mathrm{hifas} \mathrm{septadas} \mathrm{hialinas}$ con conexiones en asa. En agar Sabouraud creció un mohoblanco que por sus características macroscópicas y microscópicas se identificó comoS. commune. El paciente fue tratado con itraconazol y después de cuatro meses hubo resolución de las manifestaciones clínicas. A pesar de su baja frecuencia, este hongo se debe considerar como posible patógeno, en especial en muestras de senos paranasales.

Palabras clave: Basidiomiceto; Sinusitis; Schizophyllum commune; Diagnóstico; Itraconazol.

Schizophyllum commune is a mould of Phylum Basidiomycota, Schizophyllaceae family, with worldwide distribution that colonizes diverse trees and rotting wood $^{1}$. Infections originated from this fungus are rare in humans. Diverse clinical cases include chronic or allergic sinusitis, pulmonary disease, ulcerative lesions of the palate, atypical meningitis, cerebral abscess, and possible onychomycosis, which can occur in immunocompetent and immunocompromisedindividuals ${ }^{2,3}$. The infective propagules in this fungus are air transported, thus, most frequently compromising the paranasal sinuses ${ }^{1}$, hence, the most common affliction is sinusitis that presents three clinical manifestations: allergy, chronic non-invasive and invasive (acute or chronic) ${ }^{4}$.

In the laboratory, $S$. commune grows with relative ease in culture media that do not contain cyclohexamide; due to this characteristic, it may be suspected that it is an environmental contaminant. But upon positive direct examination and isolation of this fungus, it is necessary to carry out repeated cultures ${ }^{5}$. In the culture, the

1. Assistant Professor, School of Bacteriology and Clinical Laboratory, Universidad del Valle, Cali, Colombia. e-mail: luzacastro@yahoo.com

2. Professor,Department of Microbiology,Universidad del Valle,Cali,Colombia.e-mail:malvarez@ univalle.edu.co

3. Auxiliary Professor, Department of Internal Medicine, Universidad del Valle. Infectologist, Hospital Universitario del Valle, Cali,Colombia.e-mail:emarbui@yahoo.com

Received for publication August 26,2008 Accepted for publication January 12th, 2010 


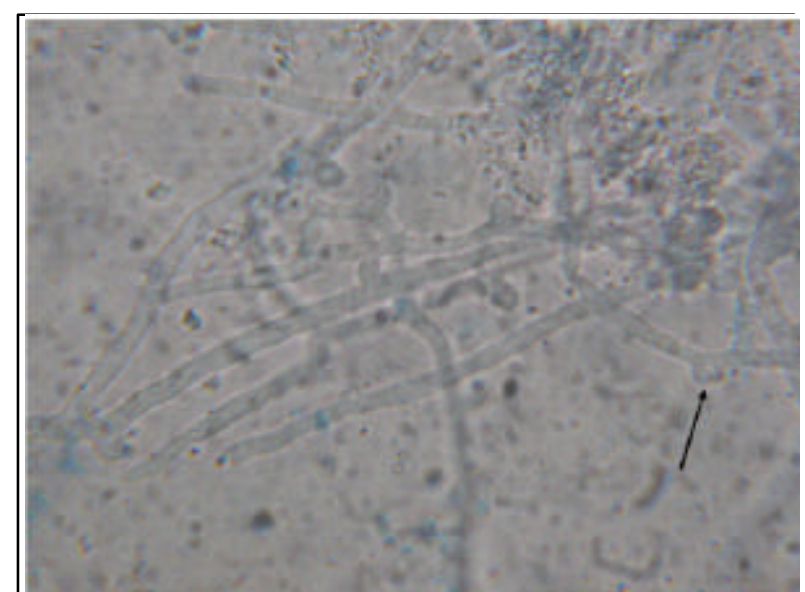

Photograph $1.20 \% \mathrm{KOH}$, showing branched septate hyaline hyphae with clamp connections (40X).

appearance of fruiting bodies, the visualization of septate hyaline hyphae with clamp connections (fibulas) and the presence of spicules facilitate identification of this basidiomycete.

We present the isolation of S. commune from material from paranasal sinuses in a patient from Cali, Colombia. The low frequency of reports on pathologies caused by this fungus motivated the presentation of the current case.

\section{CLINICAL CASE}

We report the case of a 46-year-old male patient residing in Cali with a 6-year history of recurrent sinusitis and prior surgery of paranasal sinuses, who consults complainig of constant frontal cephalea, facial pain, and 4 months of nasal discharge of progressive intensity. The patient continued with clinical manifestations in spite of treatment with various antibiotics and topical nasal steroids. Because of the persistence of the symptoms, scan was ordered and it revealed inflammatory involvement of all the paranasal sinuses predominantly on the left side, with no air fluid levels. With diagnosis of refractory chronic sinusitis to antibiotic treatment, a left maxillary sinus antrostomy was performed and from the material obtained a request was made for bacteria, mycobacteria, and fungus cultures, as well as a histopathological study.

The bacterial cultures from the two samples of secretion from the left maxillary sinus, presented growth of Serratia marcescens; the cultures for mycobacteria

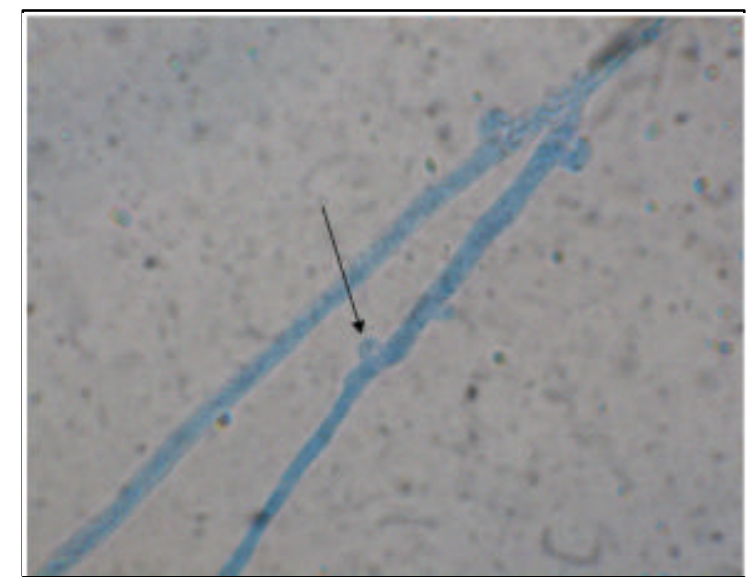

Photograph 2. Colony stained with lactophenol blue, showing hyaline hyphae with clamp connections (100x)

were negative. For analysis of fungi, directexamination and culture were performed; the direct examination with $20 \%$ potassium hydroxide $(\mathrm{KOH})$ evidenced septate hyaline hyphae, branched with clamp connections (Photograph 1). Primary cultures were done in Mycosel agar (BBL) and Sabouraud 2\% dextrose agar (BBL), incubated at $25^{\circ} \mathrm{C}$. In the first culture fungi did not grow, while in the second culture white mould developed within four days. Microscopically, this white mould revealed hyphae with the same characteristics as those observed in the direct examination (Photograph 2). After three weeks of incubation, fruiting bodies (basidiocarps) began to form (Photograph 3). Based on the clamp connections, the production of basidiocarps and the susceptibility to cyclohexamide (Mycosel agar), the fungus was identified as S. commune. The histological staining was negative for bacteria and fungi.

The patient received moxifloxacin for the bacterial infection without antifungal therapy; despite this treatment, the patient continued symptomatic without observable improvement after four weeks of antibiotic, hence, he was evaluated with a new scan of paranasal sinuses, which revealed persistent pansinusopathy (Photograph 4) and was again surgically intervened. Pus was obtained from the antrostomy, which yielded negative results for bacteria and mycobacteria; contrasting with the direct examination and culture for fungi that showed septate hyaline hyphae, branched with clamp connections that again permitted identifying S. commune. 


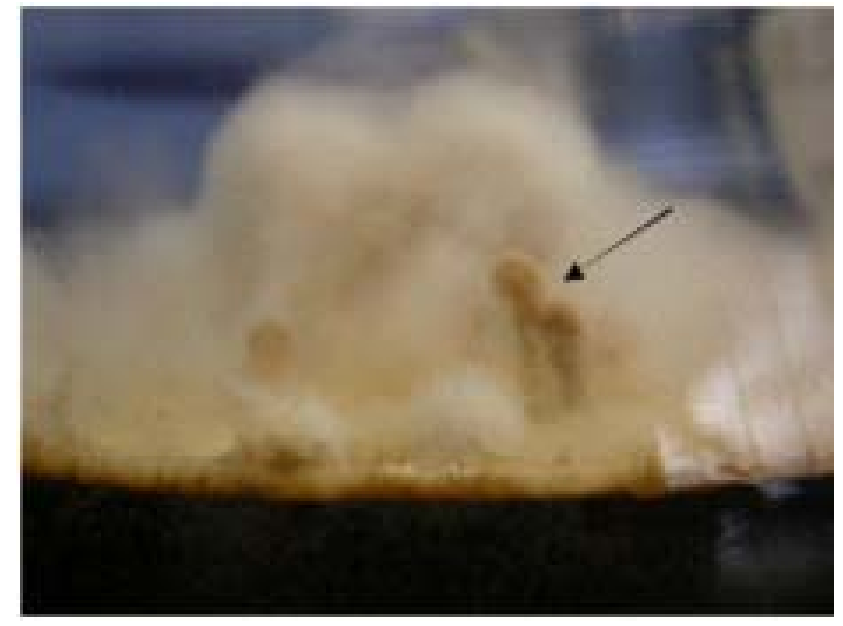

Photograph 3. Fruiting bodies (basidiocarps). Culture after 4 weeks of incubation.

For the histopathological study routine staining with hematoxylin-eosin (HE) was done, along with special staining for fungi like Periodic Acid Schiff technique (PAS) and silver-methenamine that showed the presence of hyphae with clamp connections (Photograph 5). The isolated fungus was sent to Corporación para Investigaciones Biológicas, CIB, (Corporation for Biological Research) in Medellín, for tests of minimum inhibitory concentration sensitivity and it was sensitive for amphotericin B $(0.023 \mathrm{mg} / \mathrm{ml})$, voriconazole $(0.06 \mathrm{mg} /$ $\mathrm{ml})$, itraconazole $(0.25 \mathrm{mg} / \mathrm{ml})$ and resistant to fluconazole $(>256 \mathrm{mg} / \mathrm{ml})$.

The patient was treated with itraconazole in capsules

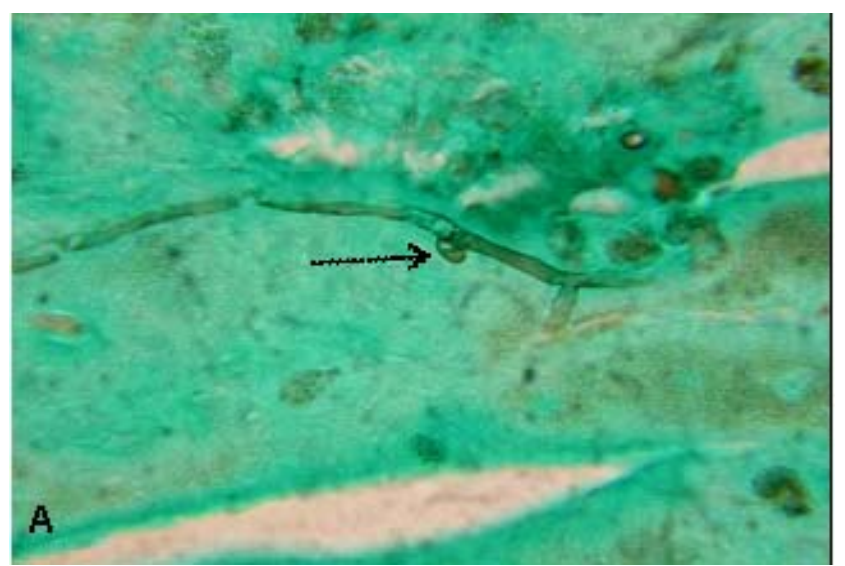

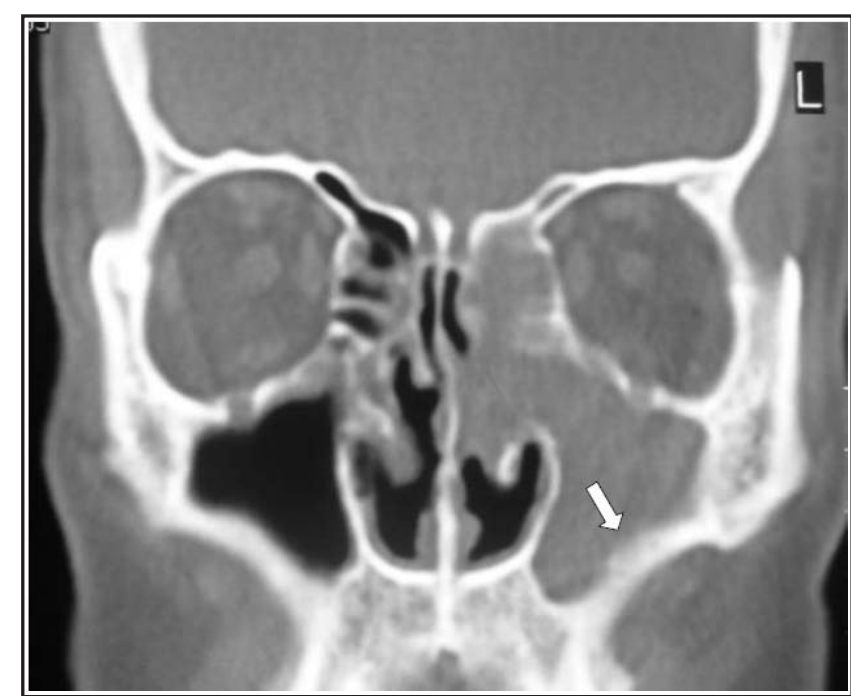

Photograph 4. Scan of left maxillary sinus prior to second isolation of $S$. commune, showing dense material (pus) and the lower floor of the sinus with thickening of the bone due to periostitis (arrow).

at a dose of $400 \mathrm{mg} /$ day for 4 months, evidencing clinical (notorious reduction of the pain symptoms, congestion, and secretion), radiologic improvement as confirmed in the control scan (Photograph 6). At the beginning of the treatment, the patient reported transient dizziness, but this did not motivate suspending the antifungal therapy. After one month with itraconazole in capsules, change was made to oral solution due to its better bioavailability. Four months of treatment were completed without side effects.

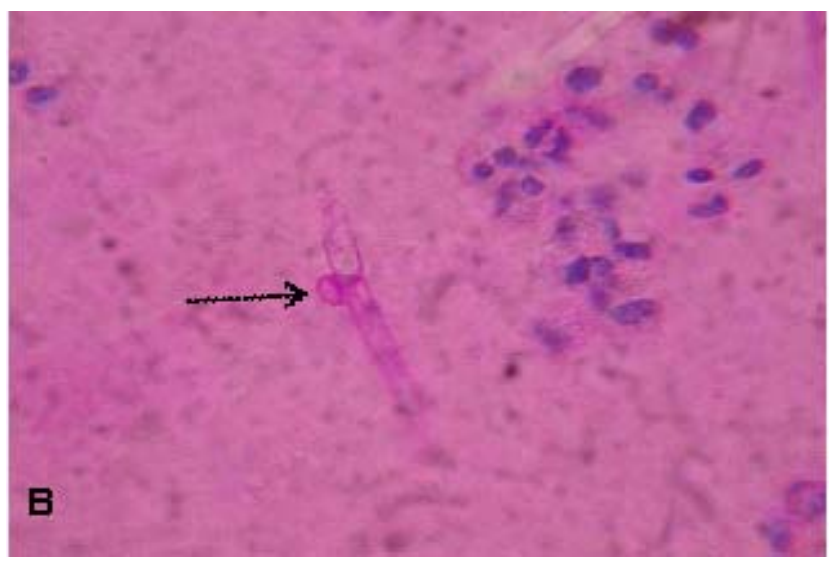

Photograph 5. Schizophyllum commune in Gomori silver-methenamine (A) and PAS staining (B) Septate hyphae with clamp connections arrows (100x) 


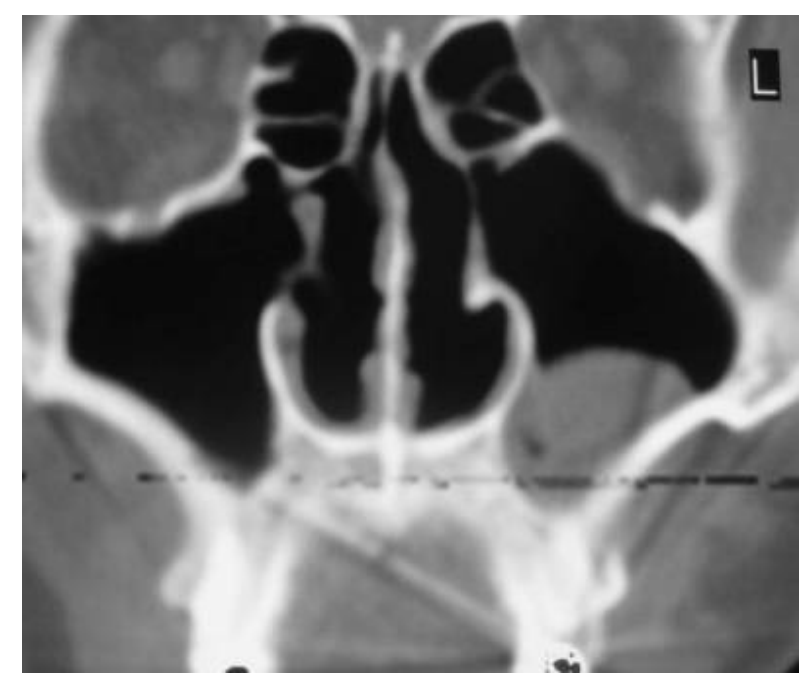

Photograph 6. Scan of posterior left maxillary sinus and after surgical debridement and 4 months of treatment with itraconazole, which shows retention cyst and improvement of inflammatory findings.

\section{DISCUSSION}

Fungal sinusitis is a rare entity that has increased in recent years in immunocompetent individuals. It reveals three clinical manifestations: allergic, chronic noninvasive and invasive. The allergic manifestation involves immunocompetent patients and it is characterized by allergic mucin with eosinophils and Charcot-Leyden crystals, as well as increased serum IgE. The chronic non-invasive manifestation affects immunocompetent individuals, and does not cause mucosal or blood vessel invasion. The invasive manifestation occurs in immunodepressed individuals with tissue and vascular invasion ${ }^{4}$.

Aspergillus is the isolated fungus with the highest frequency in invasive and non-invasive sinusitis; however, other dematiaceous fungi like Alternaria, Curvularia, and Bipolaris are reported as cause of this illness in its distinct clinical forms ${ }^{3}$.

Schizophyllum commune is infrequent as cause of pathologies; in Colombia, only two reports have been made on $S$. commune as cause of sinusitis ${ }^{4,5}$.

Despite the fact thatS. commune is a mould involved in diverse infectious processes ${ }^{2,3}$, in the current case, the initial isolation of the fungus was considered without clinical importance, due to the presence of Serratia marcescens and to the negative report for fungus of the histopathological study. The persistence of symptoms, required taking a second sample where isolating the fungus and visualizing fungal structures in the direct examination and the histopathology permitted establishing the diagnosis of non-invasive fungal sinusitis since allergic mucin with Charcot-Leyden crystals were not observed, nor significant eosinophilia, or tissue or vascular invasion ${ }^{4}$.

Intervention of the paranasal sinuses and drainage are considered the therapy of choice for maxillary fungal sinusitis. However, the optimal treatment for infections caused byS. commune is not well established, because of the few cases it is difficult to determine the adequate therapy. Some cases appearing in literature show effectiveness for the surgical procedure; others for amphotericin B or fluconazole ${ }^{4,6}$; nevertheless, the strain of the current case showed resistance to this azole with the in vitro sensitivity tests. Because the strain isolated from this patient was sensitive to itraconazole, this medication was used. Initially, only one debridement was done without clinical improvement, which was noted when performing the second surgical procedure accompanied by itraconazole for 4 months.

It is worth noting that the lack of familiarity with $S$. commune, may lead microbiologists and clinicians to consider it as a contaminant without importance, although the basidiomycetes must be kept present as potential pathogens. Given that this fungus may be isolated from the environment, the culture by itself is not a diagnostic. To attribute pathogenicity to it, its isolation must be accompanied by observation in direct examination of characteristic fungal structures. Certainty in diagnosis is greater when in addition to the positive direct examination, the organism is repeatedly isolated. Correct diagnosis will avoid unspecific treatments, chronicity of the illness, or complications to the patients.

\section{ACKNOWLEDGMENTS}

The authors express their gratitude to bacteriologist Catalina de Bedout from Unidad de Micología Médica y Experimental at Corporación para Investigaciones Biológicas (CIB) in Medellín, Colombia, for performing the sensitivity tests. 


\section{REFERENCES}

1. Restrepo A, Robledo J, Leiderman E, Restrepo M, Botero D, Bedoya VI (eds). Infecciones micóticas invasoras (IMI) causadas por patógenos infrecuentes. En: Fundamentos de Medi-

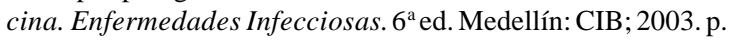
302.

2. Sigler L, Bartley JR, Parr DH, Morris AJ. Maxillary sinusitis caused by medusoid form of Schizophyllum commune. J Clin Microbiol. 1999; 37: 3395-8.

3. Taguchi K, Oharaseki T, Yokouchi Y, Kawabata T, Wakayama M, Ogoshi T, et al. Allergic fungal sinusitis caused by Bipolaris spicifera and Schizophyllum commune. Med Mycol. 2007; 45: 559-64.

4. Sigler L, Estrada S, Montealegre NA, Jaramillo E, Arango M, De Bedout C, et al. Maxilary sinusitis caused by Schizophyllum commune and experience with treatment. J Med Vet Mycol. 1997; 35: 365-70.

5. Jiménez R, Rodríguez C, Arango M, De Bedout C, Restrepo A. Sinusitis por Schyzophyllum commune: informe de nueve casos. Infectio 2002; 6:108.

6. Roan JN, Hsieh HY, Tsai HW, Wu CJ, Hsu CH, Wu SY, et al. Pulmonary nodules caused by Schizophyllum commune after cardiac transplantation. JInfect. 2009; 58: 164-7. 